\title{
Intranasal exposure of African green monkeys to SARS-CoV-2 results in acute phase pneumonia with shedding and lung injury still present in the early convalescence phase
}

\author{
Robert W. Cross ${ }^{1,2}$, Krystle N. Agans ${ }^{1,2}$, Abhishek N. Prasad ${ }^{1,2}$, Viktoriya Borisevich ${ }^{1,2}$, Courtney Woolsey ${ }^{1,2}$,
} Daniel J. Deer ${ }^{1,2}$, Natalie S. Dobias ${ }^{1,2}$, Joan B. Geisbert ${ }^{1,2}$, Karla A. Fenton ${ }^{1,2}$ and Thomas W. Geisbert ${ }^{1,2^{*}}$ (I)

\begin{abstract}
We recently reported the development of the first African green monkey (AGM) model for COVID-19 based on a combined liquid intranasal (i.n.) and intratracheal (i.t.) exposure to severe acute respiratory syndrome coronavirus 2 (SARS-CoV-2). Here, we followed up on this work by assessing an i.n. particle only route of exposure using the LMA mucosal atomization device (MAD). Six AGMs were infected with SARS-CoV-2; three animals were euthanized near the peak stage of virus replication (day 5) and three animals were euthanized during the early convalescence period (day 34). All six AGMs supported robust SARS-CoV-2 replication and developed respiratory disease. Evidence of coagulation dysfunction as noted by a transient increases in aPTT and circulating levels of fibrinogen was observed in all AGMs. The level of SARS-CoV-2 replication and lung pathology was not quite as pronounced as previously reported with AGMs exposed by the combined i.n. and i.t. routes; however, SARS-CoV-2 RNA was detected in nasal swabs of some animals as late as day 15 and rectal swabs as late as day 28 after virus challenge. Of particular importance to this study, all three AGMs that were followed until the early convalescence stage of COVID-19 showed substantial lung pathology at necropsy as evidenced by multifocal chronic interstitial pneumonia and increased collagen deposition in alveolar walls despite the absence of detectable SARS-CoV-2 in any of the lungs of these animals. These findings are consistent with human COVID-19 further demonstrating that the AGM faithfully reproduces the human condition.
\end{abstract}

Keywords: Coronavirus, SARS-CoV-2, COVID-19, Nonhuman primate, Animal models

\footnotetext{
*Correspondence: twgeisbe@utmb.edu

'Department of Microbiology and Immunology, University of Texas Medical Branch, Galveston, TX 77555, USA

${ }^{2}$ Galveston National Laboratory, University of Texas Medical Branch,

Galveston, TX 77555, USA
}

C C The Author(s). 2020 Open Access This article is licensed under a Creative Commons Attribution 4.0 International License, which permits use, sharing, adaptation, distribution and reproduction in any medium or format, as long as you give appropriate credit to the original author(s) and the source, provide a link to the Creative Commons licence, and indicate if changes were made. The images or other third party material in this article are included in the article's Creative Commons licence, unless indicated otherwise in a credit line to the material. If material is not included in the article's Creative Commons licence and your intended use is not permitted by statutory regulation or exceeds the permitted use, you will need to obtain permission directly from the copyright holder. To view a copy of this licence, visit http://creativecommons.org/licenses/by/4.0/. The Creative Commons Public Domain Dedication waiver (http://creativecommons.org/publicdomain/zero/1.0/) applies to the data made available in this article, unless otherwise stated in a credit line to the data. 


\section{Introduction}

The unprecedented pandemic of COVID-19 caused by severe acute respiratory syndrome coronavirus 2 (SARSCoV-2) has had devastating effects on public health and the global economy. Considerable resources have been allocated by governments, philanthropic organizations, and private companies in an attempt to expedite the development of vaccines and treatments to combat COVID-19. With the rapid development of 24 preventative vaccines in clinical evaluation [1], and nearly 200 more in the pipeline [2], coupled with the availability of nearly 300 candidate antivirals and disease modulators [2] it is impossible to investigate the safety and efficacy of all of these various interventions in humans. Both small animal models and nonhuman primates (NHP) may prove valuable in triaging the most promising medical countermeasures prior to use in humans. Hamsters and ferrets are currently being used as immunocompetent small animal models of COVID-19 [3-5] while several NHP models have been quickly developed [6-12]. Among the nonhuman primate models evaluated the African green monkey (AGM) appears to best recapitulate the most salient features of human COVID-19 [10-12].

We recently reported the development of the first AGM model for COVID-19 and showed that backchallenge of animals with SARS-CoV-2 5 weeks after initial exposure resulted in protection from reinfection [10]. In this study the AGMs were exposed to SARSCoV-2 by a combination of the intranasal (i.n.) and intratracheal (i.t.) routes with the virus delivered in liquid media. As a natural extension of this initial work we sought to assess the pathogenesis of SARS-CoV-2 in AGMs exposed by the i.n. route only using the LMA Mucosal Atomization Device (MAD). Previous studies with another respiratory virus, Nipah virus, showed that there were no major differences in disease pathogenesis when virus was delivered to AGMs by a combined liquid-based i.n. and i.t. delivery [13] or by the LMA MAD system [14]. The LMA MAD was developed for the efficient and safe delivery of test particles and is currently employed to administer US FDA approved drugs for i.n. delivery. The LMA MAD delivers atomized particles that range in size from 30 to $100 \mu \mathrm{m}$, which is highly consistent with the size of droplets exhaled by humans due to coughing [15]. In addition, in our previous work as the AGMs were back challenged with SARS-CoV-2 it was impossible to assess tissue pathology during convalescence after primary challenge. Here, we focused on assessing the pathogenesis of SARS-CoV-2 infection in AGMs when administered as 30 to $100 \mu \mathrm{m}$ particles and on evaluating virus shedding and lung pathology during early convalescence.

\section{Materials and methods}

\section{Virus}

The virus (SARS-CoV-2/INMI1-Isolate/2020/Italy) was isolated on January 30, 2020 from the sputum of the first clinical case in Italy, a tourist visiting from the Hubei province of China that developed respiratory illness while traveling [16]. The virus was initially passaged twice (P2) on Vero E6 cells; the supernatant and cell lysate were collected and clarified following a freeze/thaw cycle. This isolate is certified mycoplasma and Foot-andMouth Disease virus free. The complete sequence was submitted to GenBank (MT066156) and is available on the GISAID website (BetaCoV/Italy/INMI1-isl/2020: EPI_ISL_410545) upon registration. For in vivo challenge, the P2 virus was propagated on Vero E6 cells and the supernatant was collected and clarified by centrifugation making the virus used in this study a P3 stock.

\section{Animal challenge}

SARS-CoV-2 seronegative AGMs (Chlorocebus aethiops) (6 females) (St Kitts origin, Worldwide Primates, Inc.) were randomized into two cohorts where one group $(n=3)$ was scheduled for euthanasia at $5 \mathrm{dpi}$ and the other at $34 \mathrm{dpi}$. Animals were anesthetized with ketamine and inoculated with a target dose of $3.0 \times 10^{6} \mathrm{PFU}$ of SARS-CoV-2 (SARS-CoV-2/INMI1-Isolate/2020/Italy) using the LMA MAD, with the dose being equally divided between each nostril. All animals were longitudinally monitored for clinical signs of illness including temperature (measured by surgically implanted DST micro- $\mathrm{T}$ small implantable thermo loggers (Star-Oddi, Gardabaer, Iceland)), respiration quality, and clinical pathology. All measurements requiring physical manipulation of the animals were performed under sedation by ketamine. Mucosal swabs were obtained using sterile swabs inserted into the mucosal cavity, gently rotated to maximize contact with the mucosal surface, and deposited into $2.0 \mathrm{~mL}$ screw-top tubes containing sterile MEM media supplemented to $2 \%$ with FBS.

\section{RNA isolation from SARS-CoV-2-infected AGMs}

On specified procedure days (days $0,2,3,4,5,7,12,15$, $21,28,34), 100 \mu \mathrm{l}$ of blood was added to $600 \mu \mathrm{l}$ of AVL viral lysis buffer (Qiagen) for virus inactivation and RNA extraction. Following removal from the high containment laboratory, RNA was isolated from blood and swabs using the QIAamp viral RNA kit (Qiagen).

\section{Detection of SARS-CoV-2 load}

RNA was isolated from blood and mucosal swabs and assessed using the CDC SARS-CoV-2 N2 assay primers/ probe for reverse transcriptase quantitative PCR (RTqPCR) [17]. SARS-CoV-2 RNA was detected using Onestep probe $\mathrm{RT}-\mathrm{qPCR}$ kits (Qiagen) run on the CFX96 
detection system (Bio-Rad), with the following cycle conditions: $50^{\circ} \mathrm{C}$ for $10 \mathrm{~min}, 95^{\circ} \mathrm{C}$ for $10 \mathrm{~s}$, and 45 cycles of $95^{\circ} \mathrm{C}$ for $10 \mathrm{~s}$ and $55^{\circ} \mathrm{C}$ for $30 \mathrm{~s}$. Threshold cycle $\left(C_{T}\right)$ values representing SARS-CoV-2 genomes were analyzed with CFX Manager Software, and data are presented as GEq. To generate the GEq standard curve, RNA was extracted from supernatant derived from Vero E6 cells infected with SARS-CoV-2/INMI1-Isolate/2020/ Italy was extracted and the number of genomes was calculated using Avogadro's number and the molecular weight of the SARS-CoV-2 genome.

Infectious virus was quantitated by plaque assay on Vero E6 cells (ATCC CRL-1586) from all blood plasma and mucosal swabs, and bronchoalveolar lavage (BAL) samples. Briefly, increasing 10-fold dilutions of the samples were adsorbed to Vero E6 cell monolayers in duplicate wells $(200 \mu \mathrm{l})$. Cells were overlaid with EMEM medium plus $1.25 \%$ Avicel, incubated for 2 days, and plaques were counted after staining with $1 \%$ crystal violet in formalin. The limit of detection for this assay is $25 \mathrm{PFU} / \mathrm{ml}$.

\section{Hematology and serum biochemistry}

Total white blood cell counts, white blood cell differentials, red blood cell counts, platelet counts, hematocrit values, total hemoglobin concentrations, mean cell volumes, mean corpuscular volumes, and mean corpuscular hemoglobin concentrations were analyzed from blood collected in tubes containing EDTA using a Vetscan HM5 hematologic analyzer (Abaxis). Serum samples were tested for concentrations of albumin, amylase, alanine aminotransferase (ALT), aspartate aminotransferase (AST), alkaline phosphatase (ALP), blood urea nitrogen (BUN), calcium, creatinine (CRE), C-reactive protein (CRP), gamma-glutamyltransferase (GGT), glucose, total protein, and uric acid by using a Piccolo point-of-care analyzer and Biochemistry Panel Plus analyzer discs (Abaxis). Partial pressures of $\mathrm{CO}_{2}$ and $\mathrm{O}_{2}$ were obtained using an iSTAT Alinity hematological analyzer (Abbott).

\section{Serum neutralization assay}

Neutralization titers were calculated by determining the dilution of serum that reduced $50 \%$ of plaques $\left(\mathrm{PRNT}_{50}\right)$. A standard 100 PFU amount of SARS-CoV-2 was incubated with two-fold serial dilutions of serum samples for 1 hour. The virus-serum mixture was then used to inoculate Vero E6 cells for $60 \mathrm{~min}$. Cells were overlaid with EMEM medium plus 1.25\% Avicel, incubated for 2 days, and plaques were counted after staining with $1 \%$ crystal violet in formalin.

\section{ELISA}

SARS-CoV-2-specific IgG antibodies to nucleoprotein were measured in sera by ELISA at the indicated time points. Nucleoprotein ELISA kits were kindly provided by Zalgen Labs, LLC. Sera were initially diluted 1:100 and then two-fold through 1:25,600 in 4 in (1 x PBS with $0.02 \%$ Tween-20). After a one-hour incubation, plates were washed six times with wash buffer $(1 \mathrm{x}$ PBS with $0.2 \%$ Tween-20) and incubated for an hour with a 1:5000 dilution of horseradish peroxidase conjugated anti-primate IgG antibody (Fitzgerald Industries International; Cat: 43RIG020HRP). Tetramethylbenzidine was used to develop the reaction; the reaction was stopped with methane-sulfonic acid and plates were read at a wavelength of $450 \mathrm{~nm}$. Absorbance values were normalized by blank-subtracting values from wells incubated with sera from a SARS-CoV-2naive animal at the corresponding serum dilution. Endpoint titers were defined as the reciprocal of the last adjusted serum dilution with a value $\geq 0.20$.

\section{Histopathology and immunohistochemistry}

Necropsy was performed on all subjects euthanized at 5 dpi and 34 dpi. Tissue samples of all major organs were collected for histopathologic and immunohistochemical (IHC) examination and were immersion-fixed in 10\% neutral buffered formalin for $>7$ days. Specimens were processed and embedded in paraffin and sectioned at $5 \mu \mathrm{m}$ thickness. For IHC, specific anti-SARS immunoreactivity was detected using an anti-SARS nucleocapsid protein rabbit primary antibody at a 1:800 dilution for $60 \mathrm{~min}$ (Novusbio). The tissue sections were processed for IHC using the ThermoFisher Scientific Lab Vision Autostainer 360 (ThermoFisher Scientific). Secondary antibody used was biotinylated goat anti-rabbit IgG (Vector Laboratories) at 1:200 for $30 \mathrm{~min}$ followed by Vector Streptavidin Alkaline Phosphatase at a dilution of 1:200 for $20 \mathrm{~min}$ (Vector Laboratories). Slides were developed with Bio-Red (Biopath) for $7 \mathrm{~min}$ and counterstained with hematoxylin for 1 minute. For IHC, specific anti-fibrin was detected using an anti-fibrin monoclonal mouse primary antibody at a 1:3200 dilution for $60 \mathrm{~min}$ (Sekisui Diagnostics). The tissue sections were processed for IHC using the ThermoFisher Scientific Lab Vision Autostainer 360 (ThermoFisher Scientific). Secondary antibody used was biotinylated goat antimouse IgG (Vector Laboratories) at 1:200 for $30 \mathrm{~min}$ followed by Vector Streptavidin Alkaline Phosphatase at a dilution of 1:200 for $20 \mathrm{~min}$ (Vector Laboratories). Slides were developed with Bio-Red (Biopath Laboratories) for 7 min and counterstained with hematoxylin for 1 minute. Tissues were stained following package instructions for collagen with the Trichrome One-Step Blue \& Red Stain Kit (American MasterTech Scientific Laboratory Supplies).

\section{Results}

SARS-CoV-2 experimental infection of African green monkeys using the LMA MAD

We challenged six healthy, adult AGMs with a target dose of $3.0 \times 10^{6}$ PFU of SARS-CoV-2 (SARS-CoV-2/ 
INMI1-Isolate/2020/Italy) via intranasal inoculation with the LMA MAD (actual delivered dose of $2.8 \times 10^{6} \mathrm{PFU}$ ). Three animals were euthanized at 5 days post-infection (dpi) which is thought to be the approximate time point of peak disease in AGMs [10], while the remaining three animals were euthanized at 34 dpi during early convalescence. Blood and mucosal swabs were sampled from all animals on days $0,2,3,4,5$, and additionally on days 7 , $9,12,15,21,28$, and 34 for AGM-4, AGM-5, and AGM6. BAL fluid collection was performed on days $-8,3$, and 5 for all animals, as well as 7 dpi for AGM-4, AGM5 and AGM-6. Consistent with our previous report describing the development of the combined intranasal and intratracheal SARS-CoV-2 challenge model in AGMs [10], we did not observe overt signs of clinical illness in any AGMs in this study, other than decreased appetite or brief (single day) anorexia (Supp Table 1). Temperature was longitudinally monitored in $15 \mathrm{~min}$ increments for the entire study duration using surgically implanted temperature loggers; several animals (AGM-4, AGM-6) experienced brief $(<2 \mathrm{~h})$ periods of mildly elevated temperatures at $3 \mathrm{dpi}$, and two animals (AGM-2, AGM-3) exhibited an abnormal temperature cycling pattern at 3 dpi (Supp Fig. 1).

As in our previous report, transient shifts in leukocyte populations, predominately manifested as lymphocytopenia (5/6 animals), thrombocytopenia (3/6 animals), and granulocytosis (defined by neutrophilia, eosinophilia, and/or basophilia) (6/6 animals) were observed, while markers for renal (BUN, CRE) and hepatic function (ALT, AST, ALP, GGT) remained unchanged for the most part, with the exception of mild $(\leq 2$-fold) increases in ALT (2/6 animals), and mild to moderate (1 to 16-fold) increases in CRP, a marker of acute systemic inflammation (5/6 animals) (Supp Table 1), although statistical significance was not reached for most parameters at most time points (Fig. 1). In addition, hypercapnia (defined here as $\geq 4 \mathrm{mmHg}$ increase in dissolved $\mathrm{CO}_{2}$ ) was observed in 3/6 animals (Supp Table 1), which as we observed previously [10], appeared to follow a biphasic pattern (Fig. 1a, data shown as fold-change from baseline]).

All animals exhibited normal prothrombin times (PT) as compared to their individual baseline values; however, mild to moderate prolongation of the activated partial thromboplastin time (aPTT) was also observed in all animals through the acute phase of disease, most prominently in AGM-1 and AGM-2, indicating possible disorder of the intrinsic coagulation pathway (Fig. 1h, i); this was mirrored by increased levels of circulating fibrinogen (Fig. 1j). We previously showed that the pathways connected to IL- 6 production are activated during SARS-CoV-2 infection of AGMs [10], indicating possible mechanisms of coagulopathy in the current study.
All animals seroconverted, with weakly neutralizing titers (as quantified by $\mathrm{PRNT}_{50}$ ) being detected as early as $5 \mathrm{dpi}$ and gradually increasing in potency by $34 \mathrm{dpi}$, with terminal neutralizing antibody titers ranging from $\sim 1$ : 16-1:128 (Fig. 2a-e). We next quantified SARS-CoV-2 nucleoprotein specific IgG by ELISA (Fig. 2f). Seroconversion was not detected until day 15 in two animals (AGM-4 \& AGM-5). Interestingly, not until 34 dpi was a modest level $(1: 800)$ of seroconversion detected in the third animal.

\section{Quantification of viral load in blood, mucosal swabs, and lungs}

Viral RNA (vRNA) was purified from whole blood, oral, nasal and rectal mucosa, and BAL fluid from all collection days, as well as from lung tissue harvested at necropsy. As we previously reported [10], we were unable to detect SARS-CoV-2 vRNA in whole blood by RT-qPCR, nor were we able to recover infectious virus in the plasma fraction by plaque assay, confirming a lack of either cell-associated or freely-circulating virus in the peripheral blood. SARS-CoV-2 vRNA and infectious virus was detected in the nasal mucosa from all animals as early as $2 \mathrm{dpi}$, with vRNA persisting in a single animal up to 15 dpi (Fig. 3a, b). Likewise, vRNA was detected in oral swabs from all animals beginning $2-3$ dpi before falling below the limit of detection by $7 \mathrm{dpi}$, while low quantities of infectious virus $\left(1-2 \log _{10} \mathrm{PFU} / \mathrm{mL}\right)$ were only isolated from three animals (AGM-4, AGM-5, and AGM-6) (Fig. 3c, d). Remarkably, vRNA was transiently shed from the lower gastrointestinal tract up to $28 \mathrm{dpi}$ (AGM-4 and AGM-6), although infectious virus could only be recovered from the rectal swab of a single animal (AGM-3) 4-5 dpi (Fig. 3e, f). vRNA was detected in BAL fluid from 4/6 animals $3 \mathrm{dpi}$ and up to $7 \mathrm{dpi}$ in all three animals held past $5 \mathrm{dpi}$, while infectious virus was recovered from $3 / 6$ animals (Fig. 3g, h). Detectable quantities of vRNA were absent from lungs harvested during necropsy of AGMs euthanized $34 \mathrm{dpi}$, while 6-9 $\log _{10} \mathrm{GEq} / \mathrm{g}$ were detected from all three animals euthanized at 5 dpi (Fig. 3i).

\section{Gross pathology, histopathology, and immunohistochemistry}

Necropsy was performed on all animals following euthanasia, and lungs were collected for gross examination and histopathological analysis. Consistent with our previous study utilizing a combined i.n. and i.t. inoculation route [10], all AGMs displayed varying degrees of pulmonary consolidation with hyperemia and hemorrhage, characterized by depressed and patchy dark red to light pink regions (Fig. 4, arrows). In all AGMs, the most severe lesions were located in the dorsal aspects of the lower lung lobes. A board-certified veterinary pathologist 
A

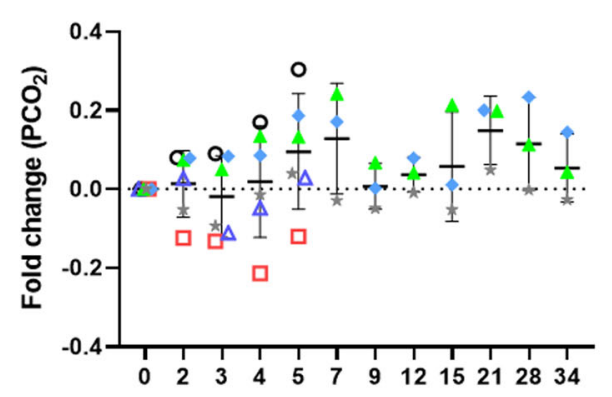

C

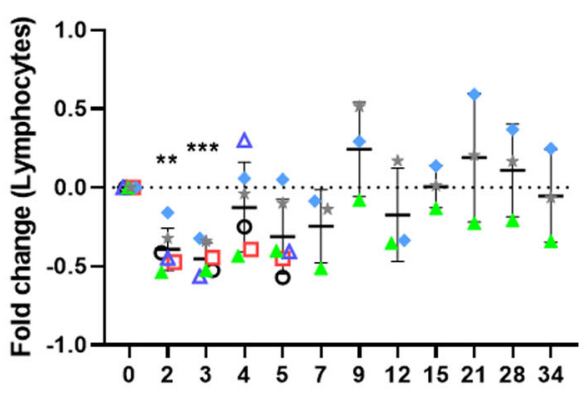

E

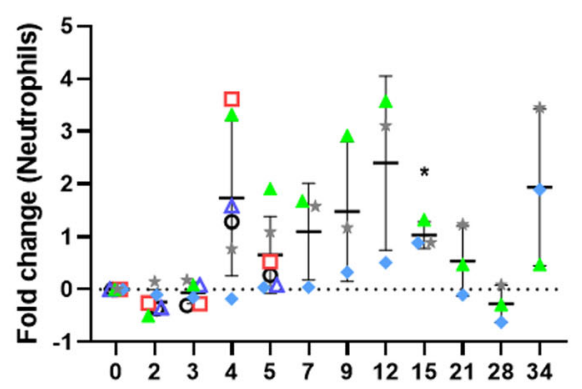

G
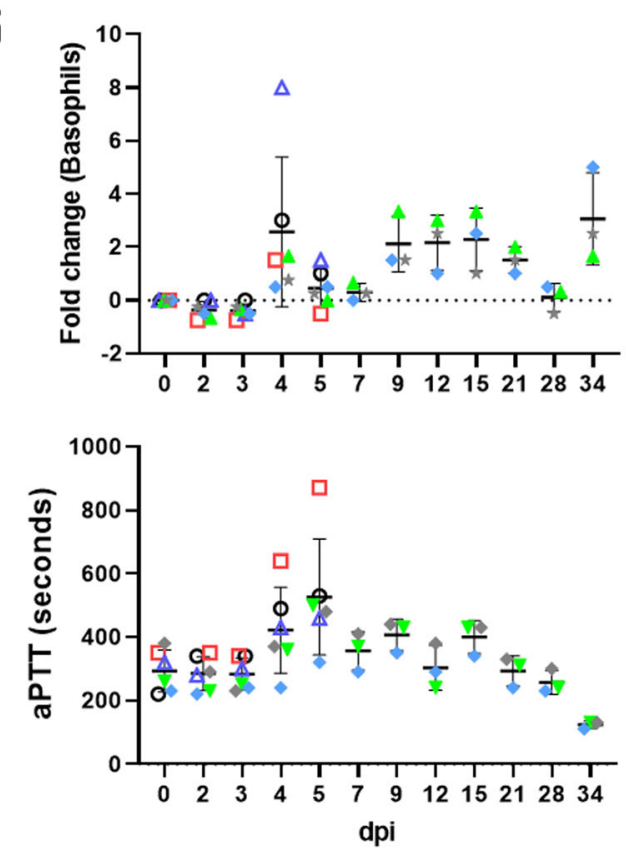

B

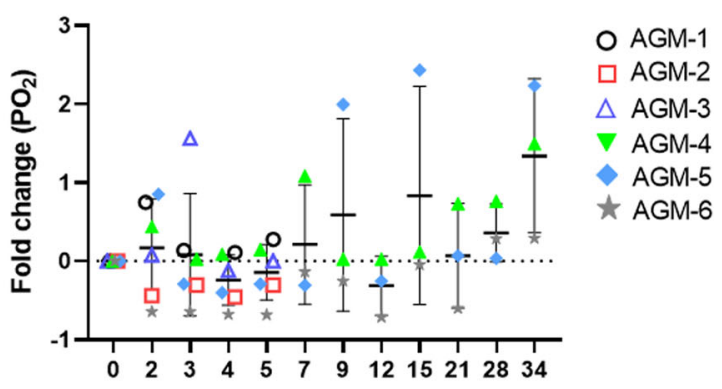

D

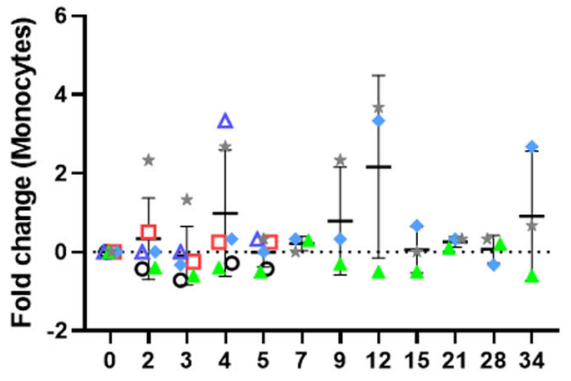

$\mathbf{F}$

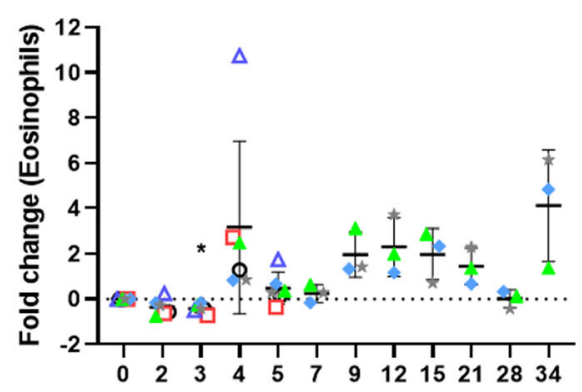

H

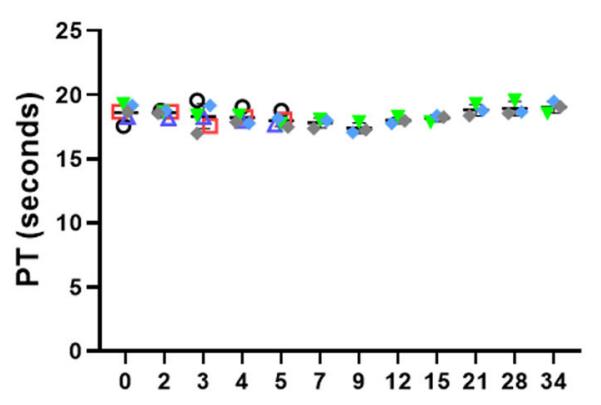

J

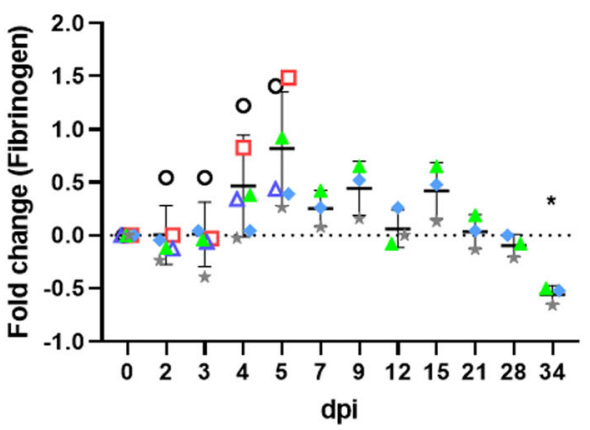

Fig. 1 (See legend on next page.) 
(See figure on previous page.)

Fig. 1 Hematological features of SARS-CoV-2 infection in AGMs. Blood gas (a, b), selected leukocyte populations (c-g), and coagulation assays (hj) are shown. For parameters where fold change is used, fold change was determined by baseline ( 0 dpi) subtraction of each time point for each animal. Statistical significance was determined in Graphpad Prism 8.4.3 by mixed-effects analysis with the Geisser-Greenhouse correction without the assumption of sphericity, with multiple comparisons made using Dunnett's post-hoc test and all comparisons made to baseline values (0 dpi). Asterisks denote significance: ${ }^{*}=p \leq 0.05,{ }^{* *}=p \leq 0.01,{ }^{* *}=p \leq 0.001$. Two-tailed $p$-values were computed for all comparisons

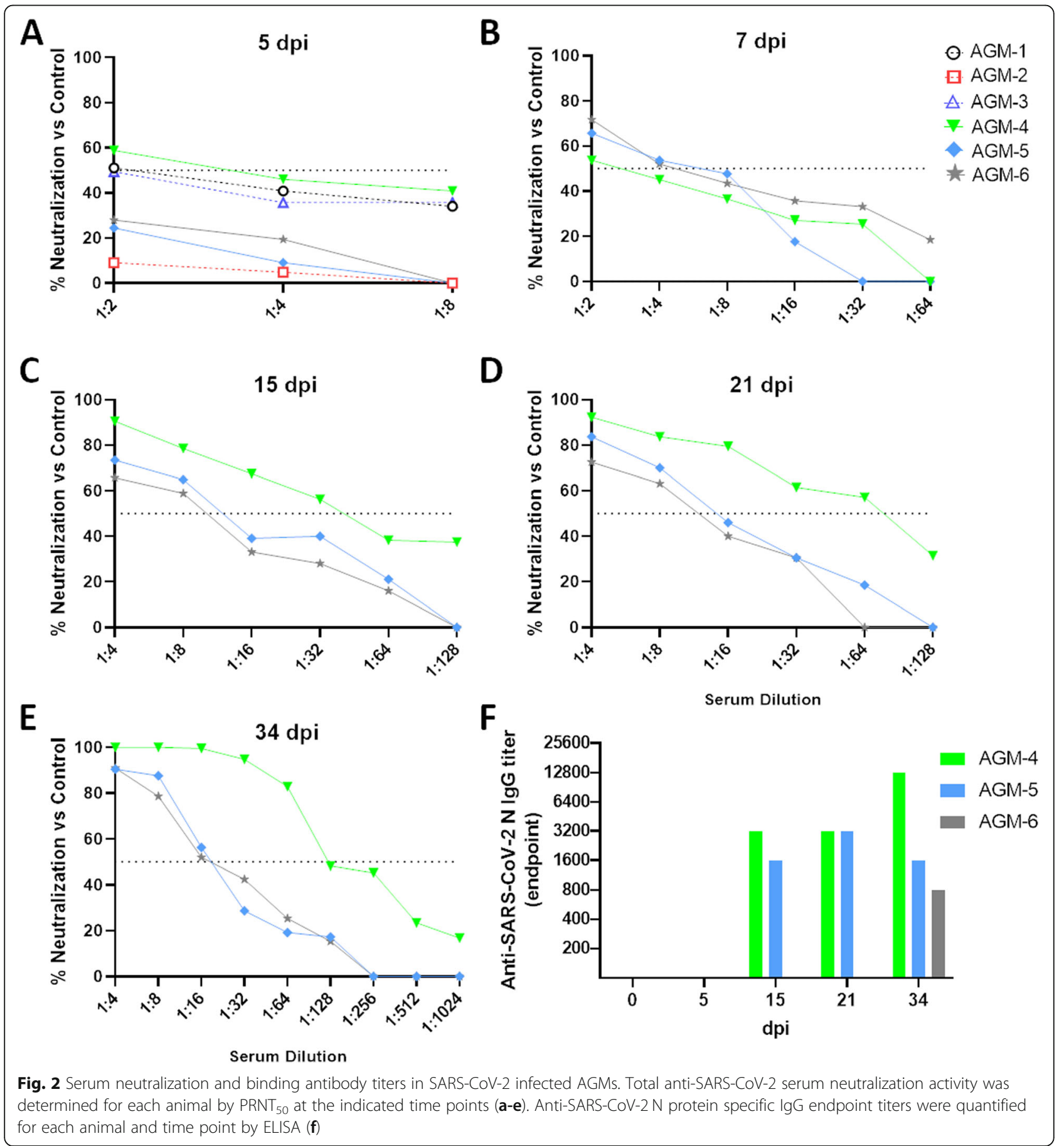




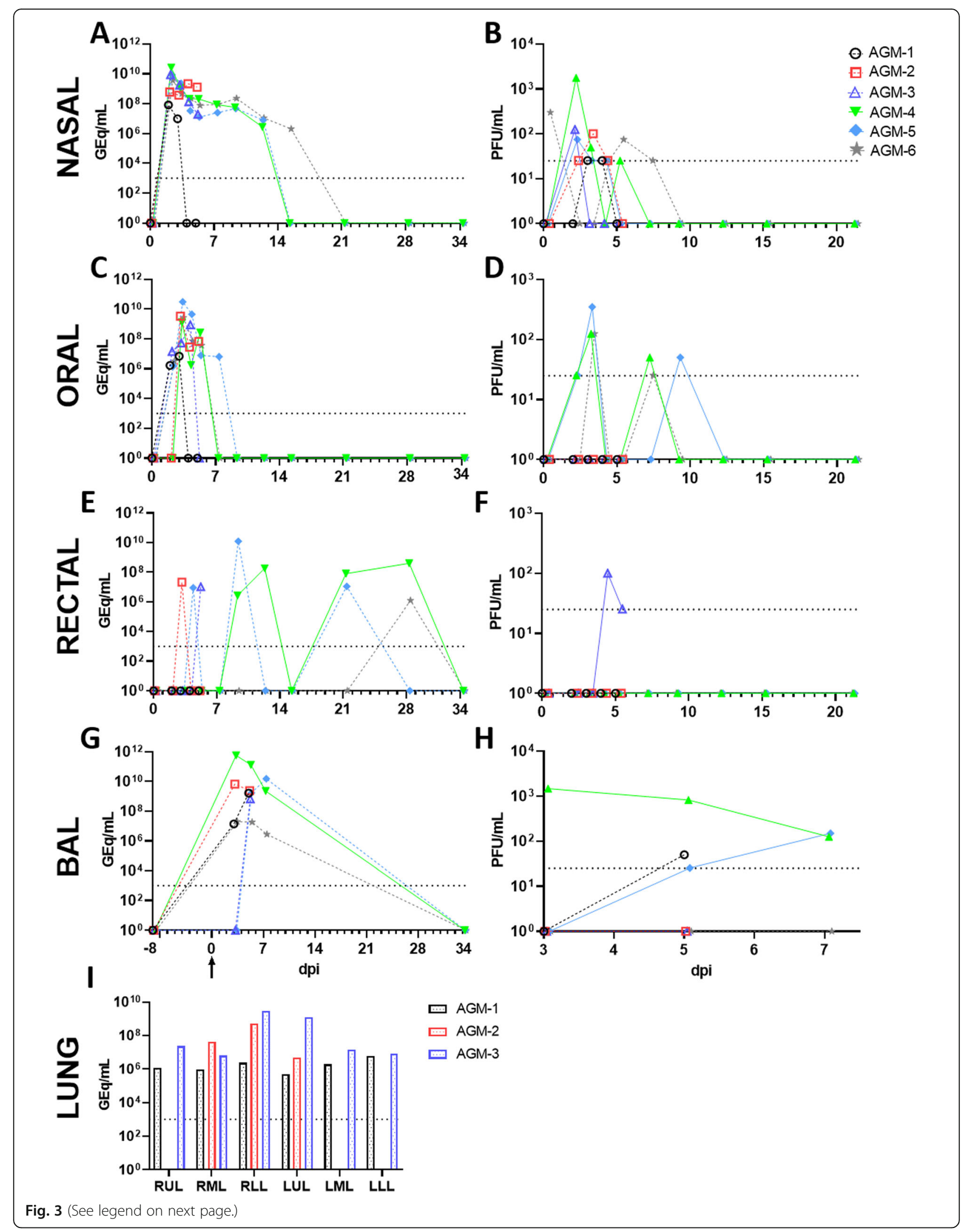


(See figure on previous page.)

Fig. 3 Quantification of SARS-CoV-2 infectious virus and VRNA in mucosal swabs, BAL fluid, and lung tissue. Viral load was quantified by detection of SARS-CoV-2 vRNA by RT-qPCR $(\mathbf{a}, \mathbf{c}, \mathbf{e}, \mathbf{g}, \mathbf{i})$ or plaque titration $(\mathbf{b}, \mathbf{d}, \mathbf{f}, \mathbf{h})$. The limit of detection for each assay is indicated by horizontal dashed line $(1000 \mathrm{GEq} / \mathrm{mL}$ for RT-qPCR, $25 \mathrm{PFU} / \mathrm{mL}$ for plaque titration). For both assays, data shown is the mean of two technical replicates of the same biological sample. Arrow in (g) indicates day of challenge. For panel I, RUL: right upper lung; RML: right middle lung; RLL: right lower lung; LUL: left upper lung; LML: left middle lung; LLL: left lower lung

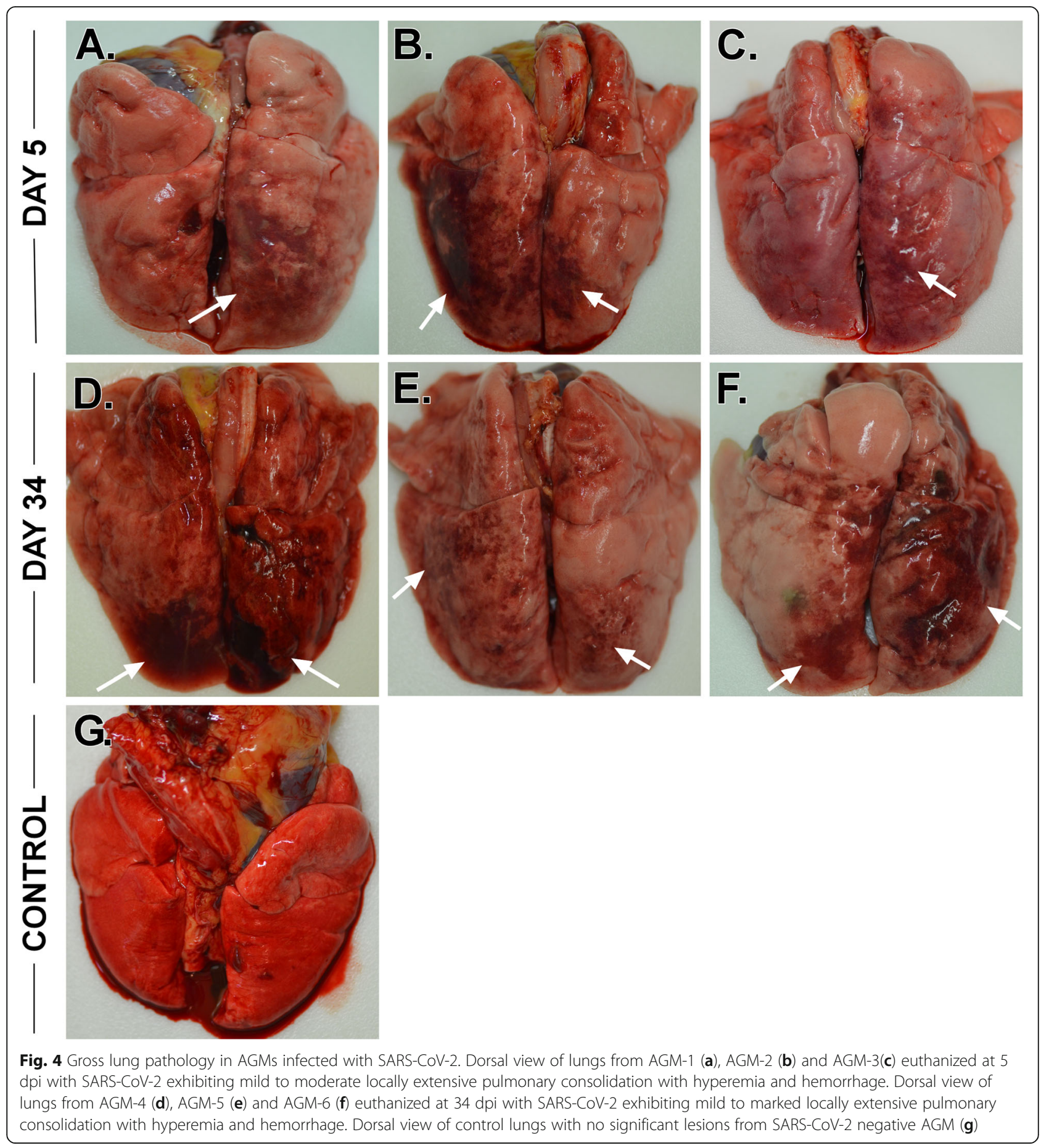




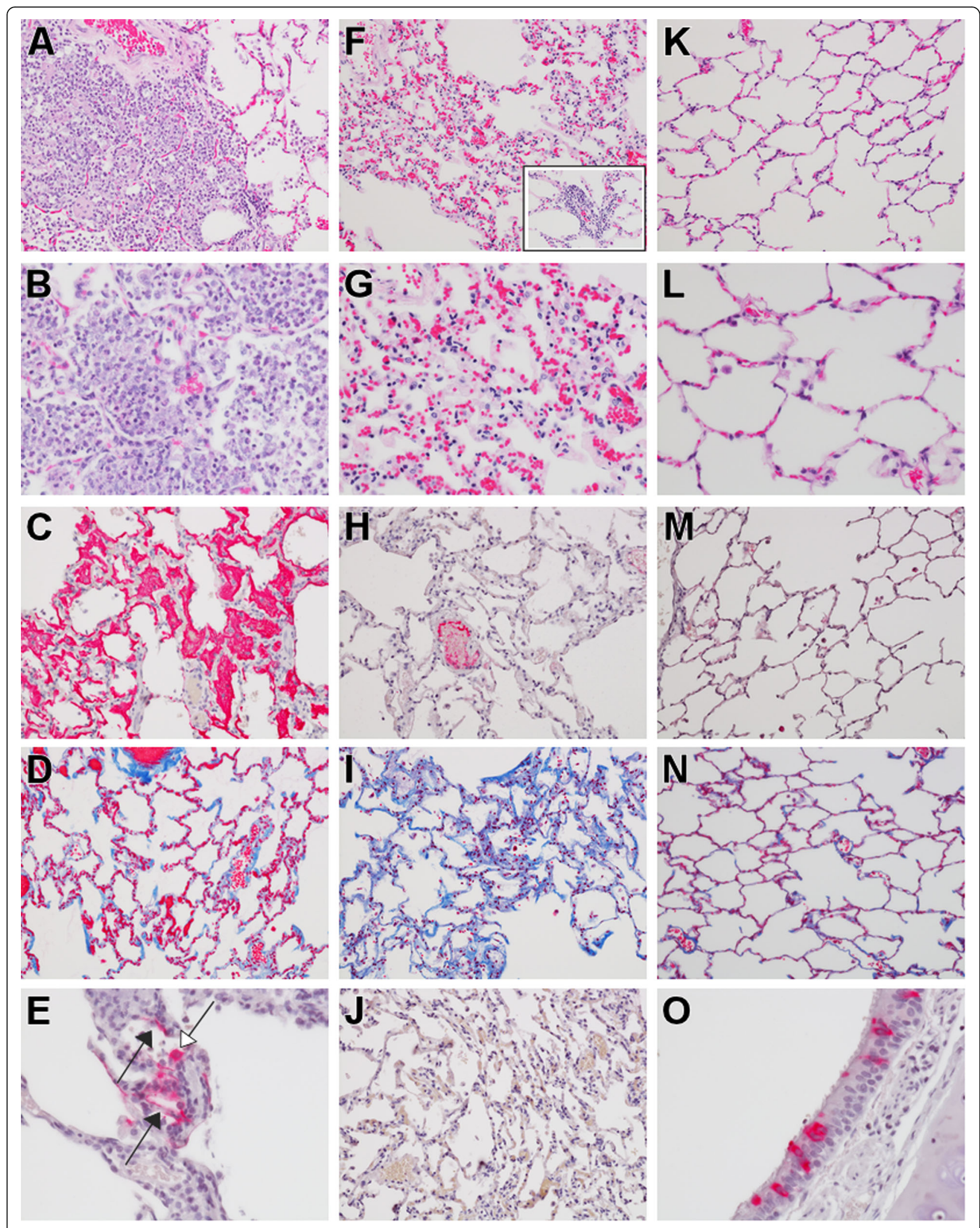

Fig. 5 (See legend on next page.) 


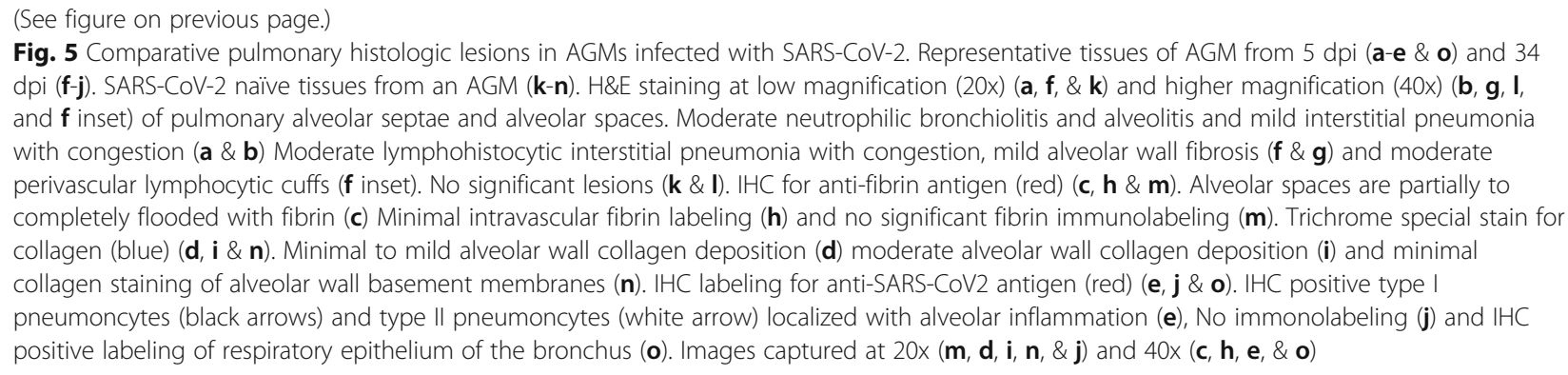

approximated lesion severity for each lung lobe (Supp Table 2). All AGMs at 5 dpi also had segmentally flaccid and gas distention of small intestines. There were no other significant gross lesions.

Histologically, all three AGMs euthanized at 5 dpi developed mild multifocal neutrophilic bronchointerstitial pneumonia (Fig. 5a-e, o). Histologic features include acute inflammation centered within the airways of terminal bronchioles with occasional flooding of adjacent alveolar spaces with neutrophils, macrophages, fibrin, edema, hemorrhage, mucous and rarely multinucleated giant cells (5A, B). In lesser-affected regions alveolar septate were expanded with mixed inflammatory cells and alveolar spaces contain increased numbers of alveolar macrophages with scattered red blood cells. Ulcerative tracheobronchitis was also present in all three AGMs and characterized by multifocal epithelial erosion associated with underlying hemorrhage, fibrin accumulation and infiltrating acute inflammation. Polymerized fibrin, highlighted by IHC, colocalized with acute inflammation within the bronchial lumen, alveolar spaces, alveolar walls and ulcerated regions of the trachea and bronchus (Fig. 5c). Fibrin was also present within medium and small caliber vessels but was not associated with an obvious adherent thrombus. Trichrome stain of representative lung sections identified modest collagen deposition within multifocal regions of alveolar septae (Fig. 5d). IHC for SARS-CoV-2 antigen was positive in all three AGMs associated with pulmonary lesions. Positive IHC labeling was noted diffusely within the cytoplasm of respiratory epithelium of the bronchus (Fig. 5o) and less in type I and type II pneumocytes (Fig. 5e).

Histologically, all three AGMs euthanized at $34 \mathrm{dpi}$ developed moderate multifocal chronic interstitial pneumonia Fig. 5f-j). Histologic features include expansion of alveolar septae with macrophages, lymphocytes, and very rarely neutrophils (Fig. 5f, g). Wispy, pale eosinophilic, acellular material also multifocally expanded the alveolar walls and stained as immature collagen with trichrome staining (Fig. 5i). Polymerized fibrin was present within medium and small caliber vessels but was not associated with an obvious adherent thrombus (Fig. 5h). No immunolabeling for SARS-CoV-2 was noted with IHC in any of the examined tissue sections from this $34 \mathrm{dpi}$ cohort (Fig. 5j).

\section{Discussion}

We previously reported the development of the AGM as a promising animal model of human COVID-19 [10]. Other studies have subsequently reported similar findings $[11,12]$. The focus of the current study was to assess a more natural route of human exposure, specifically an exposure mimicking an infection resulting from mucosal exposure to infectious droplets expelled from close quarter exposure to a sneeze, cough, or even speech in order to begin characterization of lung pathology in the early convalescence phase of COVID-19. The disease resulting from the i.n. MAD challenge was largely reflective of that observed with the combination of the i.t. and i.n. routes except it appeared to be somewhat milder in terms of length of any fever, less severe signs of pneumonia as evidenced by reduced alveolar flooding, and a lower prevalence of SARS-CoV-2 infection [10]. However, the MAD-infected AGMs still developed virus-induced pneumonia and viral shedding was detected into the early convalescence period. While it appears that inclusion of direct i.t. instillation of SARS$\mathrm{CoV}-2$ as an exposure route may result in a more severe disease in AGMs, it is also possible that animal to animal variability may have contributed to the modest difference between the studies. SARS-CoV-2 infection of humans results in a wide spectrum of disease ranging from asymptomatic to severe and fatal disease so it is not unexpected that there could be variability among AGMs as well. While the current study employed female AGMs because of animal availability at the time the work was initiated, gender did not affect the outcome when compared to similar studies [10-12].

Coagulation dysfunction is a consistent observation in human COVID-19 and has been associated with disease severity [18-22]. Here, we performed a limited analysis of blood clotting times (PT and aPTT) and circulating fibrinogen levels to begin to characterize the coagulopathy in SARS-CoV-2-infected AGMs. Transient increases in aPTT and in circulating fibrinogen levels were observed during the acute phase of infection. Increases in PT and/ 
or aPTT have been linked to severe human COVID-19 cases in some but not all studies [18-22]. However, nearly all severe COVID-19 cases have been associated with high levels of fibrinogen [20-22].

Our findings regarding lung injury in the three AGMs that were euthanized at $34 \mathrm{dpi}$ during early convalescence are consistent with the limited human COVID-19 studies that have been reported so far. For example, a recent study of fifty-seven COVID-19 patients in China was completed during the early convalescence phase, approximately 30 days after discharge [23]. The study included 40 nonsevere cases and 17 severe cases. Thirty-one patients (54.3\%) had abnormal CT findings while abnormalities were detected in the pulmonary function tests in 43 (75.4\%) of the patients. In a second human study, 21 patients recovering from COVID-19 (without severe respiratory distress during the disease course), had lung abnormalities visible on chest $\mathrm{CT}$ at 10 days after initial onset of symptoms [24]. While other studies suggest that some of the abnormalities may be resolved over time [25, 26] more research needs to be conducted in this area.

Regarding histopathology, human data is particularly sparse. One small study performed thoracoscopies with blebs resection and pleurectomies on performed on the 16th and 23rd days from symptoms onset of two patients [27]. Despite well-known pulmonary damages induced during the acute phase of COVID-19, the late-phase gross and histological changes include nonspecific chronic reparative lesions, similarly to what we have described in the AGMs at 34 dpi. Grossly in the human study, there was non-specific diffuse pulmonary congestion, edema and hemorrhagic necrosis. Histologically, the main lesions were focused on alveolar damage with mildly thickened alveolar interstitial tissues with fibrosis and mononuclear cellular infiltration (lymphocytes, plasma cells and multinucleate giant cells). Intravascular hemorrhagic thrombosis was also noted in these specimens.

In summary, we have expanded on our previous development of the combined i.n. and i.t. inoculation model of SARS-CoV-2 in AGMs. Importantly, while AGMs challenged with SARS-CoV-2 via the LMA MAD exhibited apparently milder clinical illness and disease, hallmark features from our previous study were still apparent, notably the development of viral pneumonia during the acute phase. The AGM COVID-19 model should be useful in future studies to assess disease and develop interventions that improve recovery.

\section{Supplementary information}

Supplementary information accompanies this paper at https://doi.org/10. 1186/s12985-020-01396-W.

Additional file 1: Figure S1: Longitudinal temperature monitoring of SARS-CoV-2 infected AGMs. Temperature was longitudinally monitored for all animals via surgically-implanted temperature loggers (see Materials and methods). Data shown for all animals begins 1 day prior to infection $(-1)$ and terminates in the morning of day 5 post-infection, when AGM1, AGM-2, and AGM-3 were euthanized. Periods of elevated temperature are indicated in red. Arrows denote time of challenge.

Additional file 2: Table S1: Clinical description and outcome of African green monkeys following SARS-CoV-2 challenge. Days after SARS-CoV-2 challenge are in parentheses. All reported findings are in comparison to baseline (d0) values. Decreased appetite is defined as some food but not all food consumed from the previous day. Anorexia is defined as no food consumed from the previous day. Lymphocytopenia, monocytopenia, erythrocytopenia, thrombocytopenia, neutropenia, eosinopenia, and basopenia are defined by $a \geq 35 \%$ drop in numbers of lymphocytes, monocytes, erythrocytes, platelets, neutrophils, eosinophils, and basophils, respectively. Lymphocytosis, monocytosis, neutrophilia, eosinophilia, and basophilia are defined by a $100 \%$ or greater increase in numbers of lymphocytes, monocytes, neutrophils, eosinophils, or basophils, respectively. Hyperglycemia is defined as a $100 \%$ or greater increase in levels of glucose. Hypoglycemia is defined by $a \geq 25 \%$ decrease in levels of glucose. Hypoalbuminemia is defined by $a \geq 25 \%$ decrease in levels of albumin. Hypoproteinemia is defined by $a \geq 25 \%$ decrease in levels of total protein. Hypoamylasemia is defined by $\mathrm{a} \geq 25 \%$ decrease in levels of serum amylase. Hypocalcemia is defined by $a \geq 25 \%$ decrease in levels of serum calcium. Hypercapnia was defined as having a partial $\mathrm{CO} 2>4 \mathrm{mmHg}$ over d0 baseline values. (ALT) alanine aminotransferase, (AST) aspartate aminotransferase, (ALP) alkaline phosphatase, (CRE) Creatinine, (CRP) C-reactive protein, (Hct) hematocrit, (Hgb) hemoglobin.

Additional file 3: Table S2: Gross lung lesion severity scores in AGMs infected with SARS-CoV-2

\section{Abbreviations}

APTT: Activated partial thromboplastin time; AGM: African green monkey; BAL: Bronchoalveolar lavage; COVID-19: Coronavirus Disease 2019; IHC: Immunohistochemistry; i.n.: Intranasal; i.t.: Intratracheal; MAD: Mucosal Atomization Device; PFU: Plaque forming unit; PT: Prothrombin time; SARSCoV-2: Severe acute respiratory syndrome coronavirus 2

\section{Acknowledgments}

The authors would like to thank the UTMB Animal Resource Center for veterinary support for surgery to implant temperature data loggers and husbandry support of laboratory animals and Dr. Kevin Melody for assistance with animal studies. The virus used in this publication was kindly provided by the European Virus Archive goes Global (EVAg) project that has received funding from the European Union's Horizon 2020 research and innovation program under grant agreement No 653316.

\section{Authors' contributions}

RWC and TWG conceived and designed the study. DJD, JBG, and TWG performed the SARS-CoV-2 challenge experiments. RWC, DJD, CW, JBG, and TWG performed animal procedures and clinical observations. KNA and VB performed the clinical pathology assays. VB performed the SARS-CoV-2 infectivity assays. KNA optimized and performed the PCR. NSD optimized and performed the immunohistochemistry. CW performed ELISAs. KAF performed necropsies and analysis of the gross pathology, histopathology, and immunohistochemistry. All authors analyzed the clinical pathology, virology, and immunology data. RWC, ANP, KAF, and TWG, wrote the paper. The authors had access to all of the data and approved the final version of the manuscript.

\section{Funding}

This study was supported by funds from the Department of Microbiology and Immunology, University of Texas Medical Branch at Galveston, Galveston, TX to TWG. Operations support of the Galveston National Laboratory was supported by NIAID/NIH grant UC7AI094660.

\section{Availability of data and materials}

The data supporting the conclusions of this article are included within the article. 


\section{Ethics approval and consent to participate}

All animal studies were approved by the University of Texas Medical Branch (UTMB) Institutional Animal Care and Use Committee and adhere to the NIH Guide for the Care and Use of Laboratory Animals.

\section{Consent for publication}

Not applicable.

\section{Competing interests}

The authors declare no competing interests.

Received: 27 July 2020 Accepted: 11 August 2020

Published online: 18 August 2020

\section{References}

1. World Health Organization. DRAFT landscape of COVID-19 candidate vaccines - 13 August 2020. Available from: https://www.who.int/ publications/m/item/draft-landscape-of-covid-19-candidate-vaccines.

2. Milken Institute. COVID-19 treatment and vaccine tracker; 2020. Available from: https://covid-19tracker.milkeninstitute.org/.

3. Chan JF, Zhang AJ, Yuan S, Poon VK, Chan CC, Lee AC, et al. Simulation of the clinical and pathological manifestations of coronavirus disease 2019 (COVID-19) in golden Syrian hamster model: implications for disease pathogenesis and transmissibility. Clin Infect Dis. 2020:ciaa325. https://doi. org/10.1093/cid/ciaa325.

4. Sia SF, Yan LM, Chin AWH, Fung K, Choy KT, Wong AYL, et al. Pathogenesis and transmission of SARS-CoV-2 in golden hamsters. Nature. 2020;583:834-8.

5. Richard M, Kok A, de Meulder D, Bestebroer TM, Lamers MM, Okba NMA, et al. SARS-CoV-2 is transmitted via contact and via the air between ferrets. Nat Commun. 2020;11:3496.

6. Yu P, Qi F, Xu Y, Li F, Liu P, Liu J, et al. Age-related rhesus macaque models of COVID-19. Animal Model Exp Med. 2020;3:93-7.

7. Munster VJ, Feldmann F, Williamson BN, van Doremalen N, Pérez-Pérez L, Schulz J, et al. Respiratory disease in rhesus macaques inoculated with SARS-CoV-2. Nature. 2020. https://doi.org/10.1038/s41586-020-2324-7.

8. Rockx B, Kuiken T, Herfst S, Bestebroer T, Lamers MM, Oude Munnink BB, et al. Comparative pathogenesis of COVID-19, MERS, and SARS in a nonhuman primate model. Science. 2020;368:1012-5.

9. Lu S, Zhao Y, Yu W, Yang Y, Gao J, Wang J, et al. Comparison of SARS-CoV-2 infections among 3 species of non-human primates. bioRxiv. 2020:2020.04 08.031807v2. https://doi.org/10.1101/2020.04.08.031807.

10. Woolsey C, Borisevich V, Prasad AN, Agans KN, Deer DJ, Dobias NS, et al. Establishment of an African green monkey model for COVID-19. bioRxiv. 2020:2020.05.17.100289. https://doi.org/10.1101/2020.05.17.100289.

11. Blair R, Vaccari M, Doyle-Meyers LA, Roy CJ, Russell-Lodrigue K, Fahlberg M, et al. ARDS and cytokine storm in SARS-CoV-2 infected Caribbean Vervets. bioRxiv. 2020:2020.06.18.157933. https://doi.org/10.1101/2020.06.18.157933.

12. Hartman AL, Nambulli S, McMillen CM, White AG, Tilston-Lunel NL, Albe JR, et al. SARS-CoV-2 infection of African green monkeys results in mild respiratory disease discernible by PET/CT imaging and prolonged shedding of infectious virus from both respiratory and gastrointestinal tracts. bioRxiv. 2020:2020.06.20.137687. https://doi.org/10.1101/2020.06.20.137687.

13. Mire CE, Satterfield BA, Geisbert JB, Agans KN, Borisevich V, Yan L, et al. Pathogenic differences between Nipah virus Bangladesh and Malaysia strains in primates: implications for antibody therapy. Sci Rep. 2016;6:30916.

14. Geisbert JB, Borisevich V, Prasad AN, Agans KN, Foster SL, Deer DJ, et al. An intranasal exposure model of lethal Nipah virus infection in African green monkeys. J Infect Dis. 2020;221(Supplement_4):S414-8.

15. Xie X, Li Y, Sun H, Liu L. Exhaled droplets due to talking and coughing. J R Soc Interface. 2009;6(Suppl 6):S703-14.

16. Capobianchi MR, Rueca M, Messina F, Giombini E, Carletti F, Colavita F, et al. Molecular characterization of SARS-CoV-2 from the first case of COVID-19 in Italy. Clin Microbiol Infect. 2020;26:954-6.

17. Centers for Disease Control and Prevention. Research use only 2019-novel coronavirus (2019-nCoV) real-time RT-PCR primers and probes; 2020. Available from: https://www.cdc.gov/coronavirus/2019-ncov/lab/rt-pcrpanel-primer-probes.html.

18. Chen L, Yu J, He W, Chen L, Yuan G, Dong F, et al. Risk factors for death in 1859 subjects with COVID-19. Leukemia. 2020;34:2173-83.
19. Bonetti G, Manelli F, Patroni A, Bettinardi A, Borrelli G, Fiordalisi G, et al. Laboratory predictors of death from coronavirus disease 2019 (COVID-19) in the area of Valcamonica, Italy. Clin Chem Lab Med. 2020;58:1100-5.

20. Di Minno MND, Calcaterra I, Lupoli R, Storino A, Spedicato GA, Maniscalco $\mathrm{M}$, et al. Hemostatic changes in patients with COVID-19: a meta-analysis with meta-regressions. J Clin Med. 2020;9:E2244.

21. Lin J, Yan H, Chen H, He C, Lin C, He H, et al. COVID-19 and coagulation dysfunction in adults: a systematic review and meta-analysis. J Med Virol. 2020;1-11. https://doi.org/10.1002/jmv.26346.

22. Zhu J, Pang J, Ji P, Zhong Z, Li H, Li B, et al. Coagulation dysfunction is associated with severity of COVID-19: a meta-analysis. J Med Virol. 2020. https://doi.org/10.1002/jmv.26336.

23. Huang Y, Tan C, Wu J, Chen M, Wang Z, Luo L, et al. Impact of coronavirus disease 2019 on pulmonary function in early convalescence phase. Respir Res. 2020:21:163.

24. Pan F, Ye T, Sun P, Gui S, Liang B, Li L, et al. Time course of lung changes at chest CT during recovery from coronavirus disease 2019 (COVID-19). Radiology. 2020;295:715-21.

25. Liu C, Ye L, Xia R, Zheng X, Yuan C, Wang Z, et al. Chest $C T$ and clinical follow-up of discharged patients with COVID-19 in Wenzhou City, Zhejiang, China. Ann Am Thorac Soc. 2020. https://doi.org/10.1513/AnnalsATS.2020043240C.

26. Wang Y, Dong C, Hu Y, Li C, Ren Q, Zhang X, et al. Temporal changes of CT findings in 90 patients with COVID-19 pneumonia: a longitudinal study. Radiology. 2020;296:E55-64.

27. Aiolfi A, Bruni B, Biraghi T, Montisci A, Miceli A, Baronio B, et al. Late histological findings in symptomatic COVID-19 patients: a case report. Medicine (Baltimore). 2020;99:e21046.

\section{Publisher's Note}

Springer Nature remains neutral with regard to jurisdictional claims in published maps and institutional affiliations.

Ready to submit your research? Choose BMC and benefit from:

- fast, convenient online submission

- thorough peer review by experienced researchers in your field

- rapid publication on acceptance

- support for research data, including large and complex data types

- gold Open Access which fosters wider collaboration and increased citations

- maximum visibility for your research: over $100 \mathrm{M}$ website views per year

At BMC, research is always in progress.

Learn more biomedcentral.com/submissions 